\title{
Data Analysis of Optical Emission Spectroscopy: A DC Reactive Magnetron Sputtering of Titanium Dioxide Case
}

\author{
W. Kongsri*, S. Limsuwan, S. Chaiyakun, and P. Limsuwan
}

\begin{abstract}
An alternative approach for data analysis of optical emission spectroscopy was developed to overcome the dilemma of the low emission intensities and high sensitivity of the reconstruction on small errors of the line intensities. This work comprises of data analysis of an experimental dc reactive magnetron sputtering of titanium dioxide. The purposed method was based on several works and aimed to provide accuracy improvement of the method commonly employed in commercial systems. The wavelength calibration can be carried with the ease and without having to write calibration coefficients on the EEPROM. More accurate peak finding was obtained by coupling spectroscopic raw data processing with a Lorentzian line broadening and recognizing technique. The results are displayed with the advantages of the purposed method.
\end{abstract}

Keywords - OES, wavelength calibration, spectroscopic raw data, Lorentzian lineshape, titanium dioxide

\section{Introduction}

Optical emission spectroscopy (OES) is a wellestablished, noninvasive technique of plasmas applied to identify excited and ionized species during discharge deposition [1-4]. However, OES generally measures integrated emission or emission from a limited zone of plasmas. Most diagnostic methods involve the emission spectra from atomic or molecular species in conjunction with fundamental equations which are derived from Boltzmann, Saha or other equilibrium relationships [4-5] based on coronal model [6-7].

Signature of these species [The impinging particles can be the molecules, energetic ions and neutrals] could be identified through in situ investigation using OES [8].

Several works has adopted approaches for monitoring specimens, intensity of selected lines or combinations of intensities or intensity ratios [9-15].

W. Kongsri

Faculty of Science and Technology, Valaya Alongkorn Rajabhat University under the Royal Patronage, Klong Neung, Klong Luang, Pathumthani,13180, Thailand

S. Limsuwan

Department of Physics, Faculty of Science, King Mongkut's University of Technology Thonburi, Bangkok, 10140, Thailand

S. Chaiyakun

Department of Physics, Faculty of Science, Burapha University,Chonburi, 20131, Thailand
A vast different of selected lines were chosen for a single species. This is no surprise since the differences in deposition condition such as pressure, power and deposition current and etc. [16]. However, agreement between different laboratories is often dependent on the exact plasma operating conditions.

OES is a powerful plasma measurement tool, but also very complicated analysis technique especially where emission intensity can weakling reaches the measurement system. The emission intensity of the optical wavelength being measured is a convolution of the species density, the electron distribution function and the cross-section of excitation of the optical level [17].

The emission wavelengths are accurately identified fingerprint of the species in the plasma due to the energy levels of various atoms and molecules are quite well documented.

The OES instruments are also very sensitive and high degree of accuracy for atomic analysis can only be achieved with a good calibration of the equipment and its resolution [18]. Commonly, wavelength calibration from commercial systems is based on polynomial models. It is interesting to explore an alternative curve fitting methods base on models such as Fourier or Gaussian models for exploring the possibility of accuracy improvement.

Moreover in commercial systems, the spectra are usually pre-processed with smoothing of the acquired data by filtering or other smoothing tools. This leads to observable errors into the peak finding due to the high sensitivity of the reconstruction on small errors of the line intensities. Dodt and Dinklage [19] have stated the critical of the problem and they purposed data analysis approach based on previous work by Fischer and Dose [20] which is extended by a direct modeling of spectroscopic raw data rather than an analysis of pre-analyzed line intensities.

This work purposes alternative methods for OES data analysis based on several works on wavelength calibration and analysis of spectroscopic raw data applied on experimental OES results measured from a deposition of titanium dioxide. The peaks on OES spectra are identified and spectra are reconstructed based on a Lorentzian line broadening model. The results have demonstrated the validity of the purposed method with proven advantages. on accuracy improvements as well as the convenient of use of the methods. 


\section{Experimental method}

The DC magnetron sputtering system for the study is described in detail [21], along with the experiment arrangement for depositions. Briefly the system can be described as a home built reactive DC magnetron sputtering having reactor chamber of $31 \mathrm{~cm}$ in diameter. The magnetron system cathode was constructed with cylindrical core magnet and the outer ring shaped cylindrical magnet with residual induction $(\mathrm{Br})$ of approx $14.0 \mathrm{kG}$. The target is metallic titanium disc ( $99.97 \%$ purity, Kurt J. Lesker) with $54 \mathrm{~mm}$ in diameter. Sputtering argon gas (Ar, 99.999\% purity; TIG, Thailand) was controlled flow rate of $3 \mathrm{sccm}$. The base pressure was at approx. $0.001 \mathrm{~Pa}\left(1.0 \times 10^{-5} \mathrm{mbar}\right)$. During sputtering, the sputter pressure and the discharge voltage were kept at $0.4 \mathrm{~Pa}\left(4.0 \times 10^{-3} \mathrm{mbar}\right)$ and 400 volts; respectively, for all measurements while the discharging currents were set to 500, 700 and $1000 \mathrm{~mA}$.

Optical spectra were obtained through a quartz view port using an Ocean Optics, Model USB 4000 spectrometer to gather the data over a range of wavelengths $177.25 \mathrm{~nm}$ through $886.52 \mathrm{~nm}$. Wavelength calibration was carried out with Cal2000 from Ocean optics.

The spectral line broadening based upon Lorentz model of line broadening was employed to construct the measured OES spectrum using the experimental amplitudes and width a half-power maximum [19].

Due to complicate spectral lines, only certain dominate lines in the spectrum were selected for spectrum reconstruction. Lines with sufficient intensity and peak shape; above a setting threshold, were extracted from spectrum data. In order to produce a good fit between the profiles and the experimental profiles generated from the spectrometer, close observations on the spectrum where multiple overlapping lines occur were carried out by electronically expanding the specific range of spectrum.

Peak identification was based on NIST database [18]. A Matlab code was implemented to find and extract Ti, Ar and $\mathrm{O}$ lines in the forms of element and ion according to from NIST database. Also the reconstructions of spectral lines were implemented with a Matlab code, with the results compared with PLASUS Specline version 2.13.

-Spectroscopic raw data processing with background correction for a signal with peaks by following the process: 1) estimates the background, 2) regresses the varying baseline to the window points using a spline approximation, and 3 ) adjusts the background of the input signal Y.

-Finds relevant peaks in a noisy signal by peak detection from Matlab

Three wavelength calibrations with good fitting were studied and presented as following:

The wavelength as suggested in the company was based on the relationship between pixel number and wavelength in a third-order polynomial:

$$
\lambda_{p}=I+C_{1} p+C_{2} p^{2}+C_{3} p^{3}
$$

where $\lambda p$ is the wavelength of pixel $p, \quad I$ is the wavelength of pixel $0, \mathrm{C} 1$ is the first coefficient (nm/pixel), $\mathrm{C} 2$ is the second coefficient (nm/pixel) and C3 is the third coefficient (nm/pixel).

We purposed the used of the measured wavelengths instead of using pixel number for calibration. This provided two advantages. The first advantage is measurements can be carried out with factory setting values for calibration coefficients. This gets rid of the need to write to the new calibration coefficients to EEPROM every time a new calibration is needed. Moreover, the calibration procedure requires user to perform a tedious task in identifying each pixel corresponding to the peak of the reference source. The wavelength calibration based on curve fitting to a predefined-model can be done easily on a simple computer. Secondly, calibration to different light sources for better accuracy when accessible can be carried out at convenient time since the calibration is carried out in form of data analysis with the known wavelengths of the reference light source.

\section{A. Fourier Curve Fitting}

General model n-order Fourier is in the form:

$\mathrm{f}(\mathrm{x})=\mathrm{a} 0+\mathrm{a} 1 * \cos (\mathrm{x} * \mathrm{w})+\mathrm{b} 1 * \sin (\mathrm{x} * \mathrm{w})+$ $\mathrm{a} 2 * \cos \left(2 * \mathrm{x}^{*} \mathrm{w}\right)+\mathrm{b} 2 * \sin (2 * \mathrm{x} * \mathrm{w})+\mathrm{a} 3 * \cos (3 * \mathrm{x} * \mathrm{w})+$ $\mathrm{b} 3 * \sin (3 * \mathrm{x} * \mathrm{w})+\ldots+\mathrm{an} * \cos (3 * \mathrm{x} * \mathrm{w})+\mathrm{bn} * \sin (3 * \mathrm{x} * \mathrm{w})$

The coefficients of the model can easily be obtained by numerical software such as Matlab, by fitting the set measured wavelengths to the reference wavelengths of the calibration source.

\section{B. Gaussian Curve Fitting}

General model N-order Gaussian is in the form: $\mathrm{f}(\mathrm{x})=\mathrm{a} 1 * \exp \left(-((\mathrm{x}-\mathrm{b} 1) / \mathrm{c} 1)^{\wedge} 2\right)+\mathrm{a} 2 * \exp \left(-((\mathrm{x}-\mathrm{b} 2) / \mathrm{c} 2)^{\wedge} 2\right)+$ $\mathrm{a} 3 * \exp \left(-((\mathrm{x}-\mathrm{b} 3) / \mathrm{c} 3)^{\wedge} 2\right)+\ldots+\mathrm{an} * \exp \left(-((\mathrm{x}-\mathrm{bn}) / \mathrm{cn})^{\wedge} 2\right)$

The coefficients of the model are again can easily be obtained by a numerical software such as Matlab.

Where:

$\mathrm{x}=$ the measured wavelength

$f(x)=$ the corresponding calibrated wavelength

\section{Peak finding with Lorentzian line broadening recognizing}

Lorentzian line broadening $\phi(v)=1 / \pi\left(\alpha \mathrm{L} /(v-v o) 2+\alpha \mathrm{L}^{2}\right.$

where the peak finding involves finding the peak intensity higher than the setting threshold of noisy background and then determined score similarity essentially from normalized dot-product against a specific shape (Lorentzian line shape).

\section{Results and Discussion}

Table 1 Shows comparison of wavelength calibration with different methods.

It is seen in table 1showing results from 3 wavelength calibration methods; Commercial, Fourier and Gaussian methods respectively. NIST wavelength in $\mathrm{nm}$ was obtained from National Institute of Standards and Technology, Gaithersburg, MD, U.S.A. [18]. With the calibration light source was Cal2000 from Ocean optics. The calibration light source comprises of emission spectra of two atomics; $\mathrm{Hg}$ and Ar. It can be observed from the results that for the Fourier model a reasonable calibration was reached from the $1^{\text {st }}$ order of the model. The better results than the suggested commercial method were obtained by the $2^{\text {nd }}$ order. 
Proc. of Sixth International Conference On Advances in Computing, Electronics and Electrical Technology - CEET 2016 Copyright (C) Institute of Research Engineers and Doctors. All rights reserved.

ISBN no. 978-1-63248-109-2 doi: 10.15224/ 978-1-63248-109-2-20

A good result for the Gaussian model is; however, only reached with the $7^{\text {th }}$ order. This indicates that the Fourier model might be a good choice for the wavelength calibration and the $2^{\text {nd }}$ order is sufficient.

TABLE I . SHOWS COMPARISON OF WAVELENGTH CALIBRATION WITH DifFERENT METHOdS

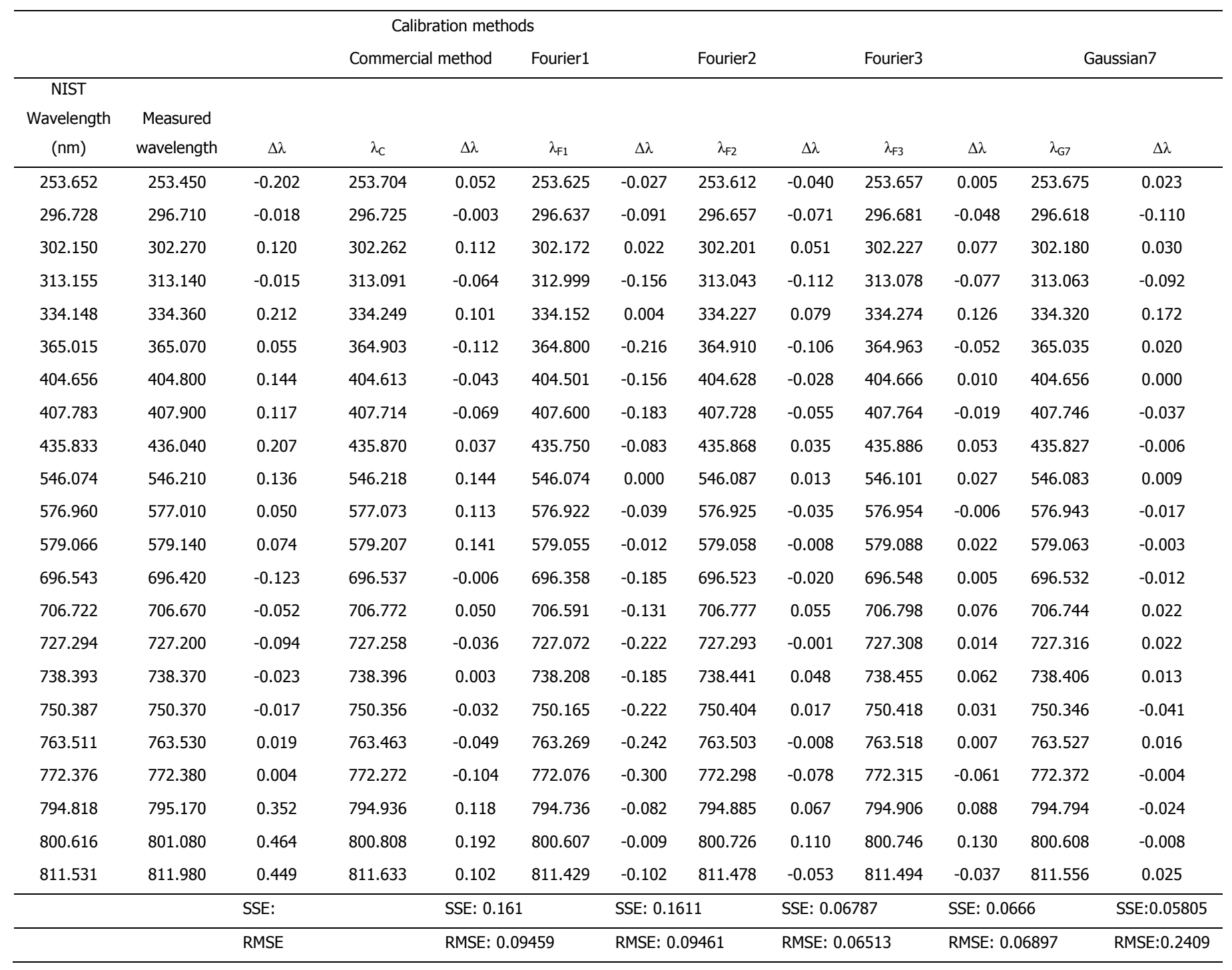

* Where $\lambda$ with subscription indicates the calibration obtained by the corresponding method and $\Delta \lambda$ is the difference from NIST data file. 
Proc. of Sixth International Conference On Advances in Computing, Electronics and Electrical Technology - CEET 2016 Copyright (C) Institute of Research Engineers and Doctors. All rights reserved.

ISBN no. 978-1-63248-109-2 doi: 10.15224/ 978-1-63248-109-2-20

Figure 1 shows a typical example of OES spectrum obtained from the sputtering of titanium dioxide using a dc magnetron sputtering system under a discharging current of $750 \mathrm{~mA}$.

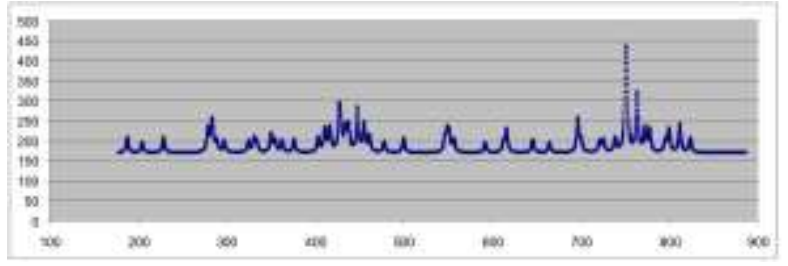

Figure 1. Shows an example of an OES spectrum of a dc magnetron sputtering of titanium dioxide at $750 \mathrm{~mA}$ under the deposition condition.

Spectroscopic raw data processing for peak finding with the Lorentzian line broadening recognizing technique was carried out and the result is shown in Figure 2.

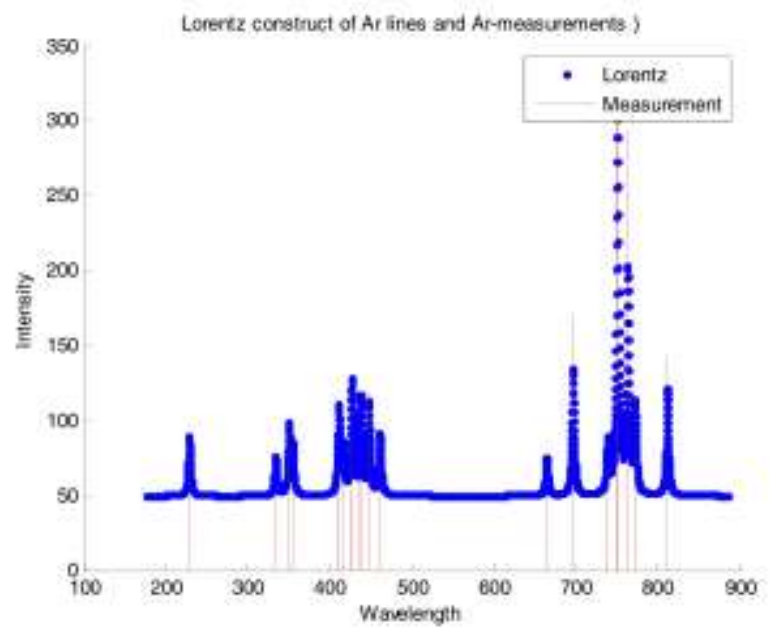

Figure 2. Shows Ar-lines of OES spectrum from a dc magnetron sputtering of titanium dioxide at $750 \mathrm{~mA}$ under the deposition condition

\section{Conclusion}

An alternative approach for data analysis of optical emission spectroscopy was developed to overcome the dilemma of the low emission intensities and high sensitivity of the reconstruction on small errors of the line intensities. The method was successfully applied to the analysis of experimental data obtained from an Oes measurement of A DC Reactive Magnetron Sputtering of Titanium Dioxide. A simpler calibration; based on Fourier curve fitting, but offers a better precision was purposed and as the result it is expected to consequently give a better accuracy on peak finding. Moreover, the method employed a spectroscopic raw data processing [20], in which the error incur from reconstruction of high sensitivity of intensity can be avoided. The peak finding and reconstructing the integrated with Lorentz line broadening modeling also offer additional accuracy to the technique as also with the good presentation of the spectra. Emission lines from atomics and ions of Arsputtering gas, O-reactive gas and $\mathrm{Ti}$ were successfully demonstrated as the results.

\section{Acknowledgment}

Wichai Kongsri would like to thank Dr. Chupong Pakphoom for the helps with various aspects on optical emission spectroscopy. This work had partially been supported by Thailand Center of Excellence in Physics (ThEP) and King Mongkut's University of Technology Thonburi under The National Research University Project.

\section{References}

1. J. Romero, and A. Lous "'OES time-resolved characterization of the deposition of multilayered $\mathrm{Cr} / \mathrm{CrN}$ coatings", Vacuum, vol. 81, pp. 1421-1425, 2007.

2. T. Makabe, and Z.Lj. Petrovic, "Development of optical computerized tomography in capacitively coupled plasma and inductively coupled plasma for plasma etching ", Applied Surface Science, vol. 192, pp. 88-114, 2002.

3. S. Martur, and P. Kuhn, "CVD of titanium oxide coatings: Comparative evaluation of thermal and plasma assisted processes", Surface \& Coatings Techonolog, vol. 201, pp. 807814, 2006.

4. R. Pintaske, Th. Welzel, M. Schaller, N. Kahl, J. Hahn, and F. Richter, "Spectroscopic studies of a magnetron sputtering discharge for boron nitride deposition", Suraace and Coatings Technolog, vol. 99, pp. 266-273, 1998.

5. B. Kullakowska-Pawlak,W.Z. yrnicki, K. Miernik, and J. Walkowicz, "Optical emission diagnostics of the linear magnetron sputtering discharge", Surface and Coatings Technology, vol. 116-119, pp. 1076-1082, 1999.

6. A.K. Sharma, R.K. Thareja, U. Willer, and W. Schade, "Phase transformation in room temperature pulsed laser deposited $\mathrm{TiO}_{2}$ thin films", Applied Surface Science, vol. 206, pp. 137-148, 2003.

7. A. De Giacomo, V.A. Shakhatov, and O. De Pascale, "Optical emission spectroscopy and modeling of plasma produced by laser ablation of titanium oxides, Spectrochimica Acta Part B, vol. 56, pp. 753-776, 2001.

8. A.B. Panda, S.K. Mahapatra, P.K. Barhai, A.K. Das, and I. Banerjee, "Understanding of gas phase deposition of reactive magnetron sputtered $\mathrm{TiO}$ thin films and its correlation with bactericidal efficiency", Applied Surface Science, vol. 258, pp. 9824-9831, 2012

9. Y. Song, T. Sakurai, K. Maruta, A. Matusita, S. Matsumto, S. Saisho, and K. Kikuchi, "Optical and structural properties of dense $\mathrm{SiO} 2$, Ta2O5 and $\mathrm{Nb} 2 \mathrm{O} 5$ thin-films deposited by indirectly reactive sputtering technique", Vacuum, vol. 59, pp. 755-763, 2000.

10. M. Audronis, and V. Bellido-Gonzalez, "Control of reactive high power impulse magnetron sputtering processes", Surface \& Coatings Technology, vol. 205, pp. 3613-3620, 2011.

11. M.Kakati, B. Bora, U.P. Deshpande, D.M. Phase, V. Sathe, N.P. Lalla, T. Shripathi, S. Sarma, N.K. Joshi, and A.K. Das, "Study of a supersonic thermal plasma expansion process for synthesis of nanostructured $\mathrm{TiO}_{2}$ ", Thin Solid Films, vol. 518, pp. 84-90, 2009.

12. I. Swindells, P.J. Kelly, and J.W. Bradley, "Phase transformation on room temperature pulsed laser deposted $\mathrm{TiO}_{2}$ thin films", Surface \& Coatings Technology, vol. 203, pp. 391-395, 2008.

13. G.S. Chen, C.C. Lee, H. Niu, W. Huang, R. Jann, T. Schutte, "Sputter deposition of titanium monoxide and dioxide thin films with controlled properties using optical emission spectroscopy", Thin Solid Films, vol. 516, pp. 8473-8478, 2008.

14. Z. Weiss, and K. Marshall, "Elemental depth profiling of coated and surface-modified materials by GD-OES: hard coatings on cutting tools", Thin Solid Films, vol. 308-309, pp. 382-388, 1997.

15. V.N. Tsaneva, Tsv.K. Popov, F.M. Dias, E.J. Tarte, M.G. Blamire, J.E. Evetts, and Z.H. Barber, "Optical emission spectroscopy and Langmuir probe characterisation of the plasma during high-pressure sputter deposition of high-Tc superconducting $\mathrm{Yba}_{2} \mathrm{Cu}_{3} \mathrm{O}_{7}-\mathrm{x}$ thin film", Vacuum, vol. 69, pp. 261-266, 2003.

16. B. Rosenkranz, and J. Bettmer, "Microwave-induced plasmaoptical emission epectrometry - fundamental aspects and 
Proc. of Sixth International Conference On Advances in Computing, Electronics and Electrical Technology - CEET 2016 Copyright (C) Institute of Research Engineers and Doctors. All rights reserved.

ISBN no. 978-1-63248-109-2 doi: 10.15224/ 978-1-63248-109-2-20

apllications in metal speciation analysis", Trends in analytical chemistry, vol. 19, pp.138-156, 2000.

17. S.C. Pyun, J.H. Kwon, S.J. You, D.J. Seong, J.H. Kim, Y.H. Shin, J.S. Shin, "On monitoring of gas leak in the plasma vacuum process with optical emission spectroscopy", vol.518, pp 66586662,2010 .

18. A. Kramida, Yu. Ralchenko, J. Reader and NIST ASD Team (2013), NIST Atomic Spectra Database (ver. 5.1) [Online]. Available: http://physics.nist.gov/asd [2014, April 23]. National Institute of Standards and Technology, Gaithersburg, MD.

19. A. Anand, J. Roberts, R. Croley Jai, and N. Dahiva, "Microwave Initiated Atomic Spectra from Select Atomic Species", Journal of Microwave Power and Electromagnetic Energy, vol. 44 (3), pp. 153-162, 2010.

20. D. Dodt and A. Dinklage, R. Fischer,and V. Dose, "Analysis of Optical Emission Spectroscopy on Discharges", MaxEnt 2006, Twenty sixth International Workshop on Bayesian Inference and Maximum Entropy Methods in Science and Engineering CNRS, Paris, France, July 8-13, 2006.

21. N. Witit-Anun, P. Kasemanankul, S. Chaiyakun, A. Pokaipisit, and P. Limsuwan, "Commparison of Nanocrystalline $\mathrm{TiO}_{2}$ Films Prepared on Unheated on Unheated Substrates Using Single- and Dual-Cathode DC Unbalanced Magnetron Sputtering Systems", Japanese Journal of Applied Physics, vol. 49, p. 051101, 2010.

About Author (s):

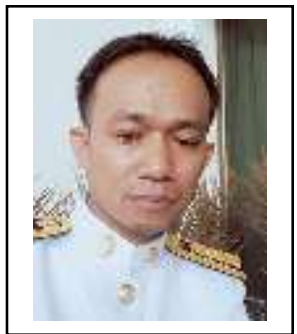

Wichai Kongsri received B.Eng from Griffith University, Australia, in 1997. He received Ms. (Physics) from King Mongkut's University of Technology Thonburi. His research interest is in material science and applications of signal processing

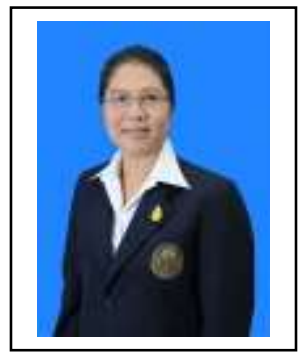

Supanee Limsuwan received her BS degree in Physics from Khon Kaen University, Thailand, in 1977 and MS degree in Physics from University of Oregon, U.S.A., in 1986. She is an associate professor of physics at the department of physics, King Mongkut's University of Technology Thonburi (KMUTT), Bangkok Thailand. Her research interests include gemology and archaeology.

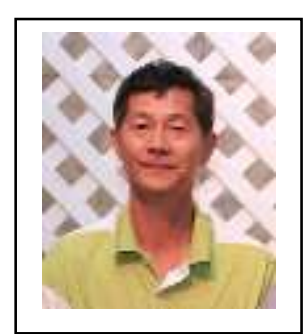

Mr. Surasing Chaiyakun received his BS degree in Physics from Burapha University, Thailand, in 1982 and MS degree in Physics from Chulalongkorn University, Thailand, in 1985. He is currently an associate professor of physics at Burapha University, Chonburi, Thailand. His research interests include vacuum and thin film technologies.

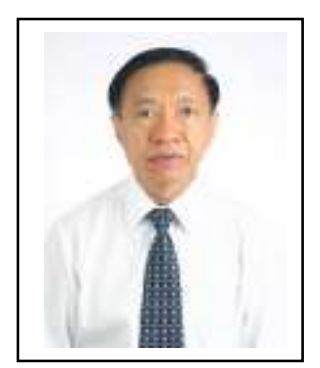

Pichet Limsuwan received his BS degree in physics from Chulalongkorn University, Bangkok, Thailand, in 1969 and PhD degree in Physics from The Pennsylvania State University, U.S.A., in 1978 . He is currently a professor of physics, the department of physics, King Mongkut's University of Technology Thonburi (KMUTT), Bangkok Thailand. His research interests include optics, lasers, materials, and thin film technology. 